\title{
Electron Transfer and Collision Induced Dissociation of Non-Derivatized and Derivatized Desmosine and Isodesmosine
}

\author{
Sara Ongay, ${ }^{1}$ Jos Hermans, ${ }^{1}$ Andries P. Bruins, ${ }^{1}$ Adrianus M. C. H. Nieuwendijk, ${ }^{2}$ \\ Hermen Overkleeft, ${ }^{2}$ Rainer Bischoff ${ }^{1}$ \\ ${ }^{1}$ Department of Analytical Biochemistry, University of Groningen, Groningen, The Netherlands \\ ${ }^{2}$ Department of Bio-Organic Synthesis, Leiden University, Leiden, The Netherlands
}

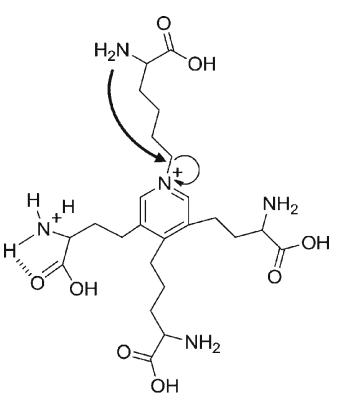

Abstract. Electron transfer dissociation (ETD) has attracted increasing interest due to its complementarity to collision-induced dissociation (CID). ETD allows the direct localization of labile post-translational modifications, which is of main interest in proteomics where differences and similarities between ETD and CID have been widely studied. However, due to the fact that ETD requires precursor ions to carry at least two charges, little is known about differences in ETD and CID of small molecules such as metabolites. In this work, ETD and CID of desmosine (DES) and isodesmosine (IDS), two isomers that due to the presence of a pyridinium group can carry two charges after protonation, are studied and compared. In addition, the influence of DES/IDS derivatization with propionic anhydride and polyethyleneglycol (PEG) reagents on ETD and CID was studied, since this is a common strategy to increase sensitivity and to facilitate the analysis by reversed-phase chromatography. Clear differences between ETD and CID of nonderivatized and derivatized-DES/IDS were observed. While CID is mainly attributable to charge-directed fragmentation, ETD is initiated by the generation of a hydrogen atom at the initial protonation site and its subsequent transfer to the pyridinium ring of DES/IDS. These differences are reflected in the generation of complex $\mathrm{CID}$ spectra dominated by the loss of small, noninformative molecules $\left(\mathrm{NH}_{3}, \mathrm{CO}, \mathrm{H}_{2} \mathrm{O}\right)$, while ETD spectra are simpler and dominated by characteristic side-chain losses. This constitutes a potential advantage of ETD in comparison to CID when employed for the targeted analysis of DES/IDS in biological samples.

Key words: Electron transfer dissociation, Collision-induced dissociation, Desmosine, Mass spectrometry, Polyethyleneglycol

Received: 2 July 2012/Revised: 15 September 2012/Accepted: 16 September 2012/Published online: 27 November 2012

\section{Introduction}

A lthough collision induced dissociation (CID) is the most common ion activation technique employed in tandem mass spectrometry, several new methods, such as surface induced dissociation (SID), electron capture dissociation (ECD), and infrared multiple-photon dissociation (IRMPD) have been developed in the last years [1]. These new techniques have been examined and their differences, advantages and disadvantages compared to CID [2-5]. Among them, ECD [6] and electron transfer dissociation (ETD) [7] have proven to be complementary techniques to the more

Electronic supplementary material The online version of this article (doi:10.1007/s13361-012-0504-x) contains supplementary material, which is available to authorized users.

Correspondence to: Rainer Bischoff; e-mail: r.p.h.bischoff@rug.nl widely used CID [8] in proteomic applications. In ECD and ETD fragmentation occurs as a consequence of the transfer of an electron to the parent ion in the gas phase, while fragmentation is initiated by collision of the parent ion with a neutral gas in CID. The advantages of ECD and ETD stem from the radical-driven fragmentation mechanism that leads to preferential cleavage of disulfide and $\mathrm{N}-\mathrm{C}_{\alpha}$ bonds in polypeptides [9, 10], without energy dissipation throughout the molecule prior to fragmentation. As a result, labile posttranslational modifications such as phosphorylations or and glycosylations are generally retained, permitting their localization, which is often impossible with CID [11-13].

ECD and ETD mechanisms of peptides have been extensively studied [14-18]. ECD uses free low-energy electrons, while in ETD electrons are transferred from an anionic electron donor molecule having a low electron binding energy (i.e., fluoranthene). In spite of these differences, both show quite 
similar peptide fragmentation patterns and yields [17]. While ECD and ETD are mainly employed for peptide fragmentation there are few studies in which ECD has been used to fragment other molecules, such as poly(alkene) glycols [19], oligodeoxynucleotides [20], and phosphate-containing metabolites [21], and to elucidate their respective fragmentation patterns. More recently, ETD has proven to be useful for the structural characterization of oligosaccharides [22] and glycerophosphocholine lipids [23]. ETD of small molecules such as metabolites has received little attention because of ETD requires precursor ions that carry at least two positive charges. In this paper, ETD and CID of desmosine (DES) and isodesmosine (IDS), two isomeric pyridinium-based amino acid analogs that act as cross-linkers within human elastin [24] (Scheme 1a), are compared. DES and IDS are of interest since their release as free amino acids or as part of DES- or IDS-containing peptides into body fluids such as sputum, plasma, or urine have been used to estimate levels of elastin degradation $[25,26]$. This is of particular interest in diseases such as chronic obstructive pulmonary disease (COPD) where degradation of elastincontaining lung tissue accompanies progression of the disease towards emphysema. DES/IDS measurement in body fluids is, thus, a potential indicator of elevated elastin turnover in the lung and a marker of the effectiveness of agents with the potential to reduce elastin breakdown $[27,28]$.

The existence of a permanently charged pyridinium group and primary amine groups within these analytes (Scheme 1a) results in multiply charged ions upon positive electrospray ionization (ESI). Notably, the doubly charged ion is observed, allowing the comparison of ETD and CID pathways. Since derivatization of DES/IDS is often used to increase retention on C18 stationary phases as well as to enhance ESI ionization efficiency, we studied ETD and CID of derivatized and nonderivatized DES/IDS. DES/IDS were derivatized with polyethyleneglycol (PEG) based on our finding that derivatization of amino acids with PEG increases ionization efficiency by a factor 10-500 ([29] and unpublished results). In addition, DES/IDS were derivatized with propionic anhydride, as this approach has been employed for the analysis of DES/IDS in urine [30].
Comparative studies between derivatized and non-derivatized DES/IDS allowed us to gain a better understanding of the ETD and CID mechanisms.

\section{Experimental Chemicals and Reagents}

DES and IDS standards were purchased from Elastin Products Inc. (Owensville, MO, USA). Stock solutions (5 $\mathrm{mM}$ ) were prepared in ultrapure water containing $0.1 \%$ formic acid (Sigma Aldrich). These solutions were kept at $-20{ }^{\circ} \mathrm{C}$ till use. Propionic anhydride (Sigma Aldrich) was employed as received. $N$-hydroxysuccinimide activatedPEGs [shortPEG: MeO-EPr-NHS (1 ethylene oxide unit); and longPEG:MeO-dPEG(8)-NHS (7 ethylene oxide units)] were purchased from Iris Biotech $\mathrm{GmbH}$ (Marktrewitz, Germany). For their use, the products were dissolved in acetonitrile (HPLC supra gradient grade, Biosolve), divided into $1 \mu \mathrm{mol}$ portions, vacuum dried and stored at $-80^{\circ} \mathrm{C}$ until use. Pentafluorophenyl-activated PEG (mediumPEG, 4 ethylene oxide units) esters containing zero or five ${ }^{13} \mathrm{C}$ atoms (unlabeledmediumPEG, ${ }^{13} \mathrm{C}_{5}$-mediumPEG) were synthesized as described previously [29], dissolved in acetonitrile, divided into $1 \mu \mathrm{mol}$ portions, vacuum dried and stored at $-80^{\circ} \mathrm{C}$ until use.

\section{DES and IDS Derivatization and LC/MS Analysis}

DES/IDS $(10 \mu \mathrm{L}$ from $5 \mathrm{mM}$ stock solutions) and the derivatization reagent $(1 \mu \mathrm{mol}$ of activated PEG or $1 \mu \mathrm{L}$ propionic anhydride $(7.8 \mu \mathrm{mol})$, Scheme $1 \mathrm{~b})$ were dissolved in $0.1 \mathrm{~mL}$ sodium phosphate buffer $\mathrm{pH}$. This mixture was left to react at $40{ }^{\circ} \mathrm{C}$ for $20 \mathrm{~min}$ in a thermomixer at $450 \mathrm{rpm}$. The reaction was followed by LC-MS. The analytical LC/MS system consisted of an Agilent series 1100 capillary LC system (Waldbronn, Germany) comprising a degasser, a binary pump with stream splitter, and flow controller, a thermostated autosampler $\left(4^{\circ} \mathrm{C}\right)$, and a thermostated column compartment $\left(40{ }^{\circ} \mathrm{C}\right)$. Derivatized DES/IDS was chromatographically separated with an Atlantis dC18 column (Waters, Etten-Leur, (a)

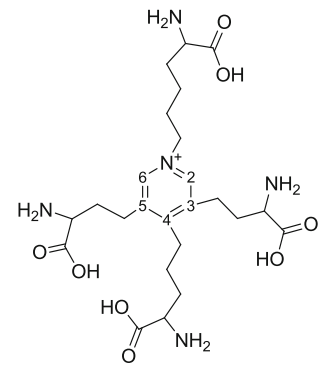

(b)

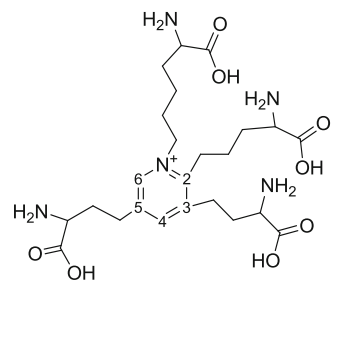

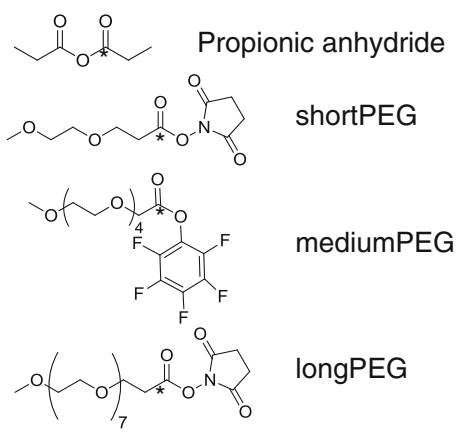

Scheme 1. (a) Chemical structures of desmosine (DES, left) and isodesmosine (IDS, right). (b) Chemical structures of the derivatization reagents, from top to bottom: propionic anhydride, shortPEG-NHS (MeO-EPr-NHS), mediumPEG-OPFP (pentafluorophenyl-activated PEG), longPEG-NHS (MeO-dPEG(8)-NHS) employed for DES/IDS derivatization. The asterisk indicates the position where the reagent binds to DES/IDS amino groups 
The Netherlands; $1.0 \mathrm{~mm} \times 150 \mathrm{~mm}$, particle size $3 \mu \mathrm{m}$ ). Mobile phase A consisted of $0.1 \%$ (vol $/ \mathrm{vol})$ formic acid in ultrapure water. Mobile phase B was $0.1 \%$ (vol/vol) formic acid in acetonitrile (HPLC-S gradient grade; Biosolve, Valkenswaard, The Netherlands). Injection volumes were $10 \mu \mathrm{L}$. The separation was performed with an increasing gradient of B $(5 \%-80 \%$ at $2 \% \times \mathrm{min}^{-1}$ ) at a flow rate of $50 \mu \mathrm{L} / \mathrm{min}$. The mass spectrometer was an Agilent SL ion trap mass spectrometer equipped with an ESI source operated in positive mode. Data were acquired over a scan range of $m / z$ 200-2200.

\section{MS/MS Analysis}

DES and IDS derivatives were fractionated, vacuum dried, and reconstituted in $20 \%(\mathrm{vol} / \mathrm{vol})$ acetonitrile, $0.1 \%$ (vol/ $/ \mathrm{vol})$ formic acid in ultrapure water to $10 \mu \mathrm{M}$ in the case of the propionylated DES/IDS derivatives and $5 \mu \mathrm{M}$ for pegylated DES/IDS. Different concentrations of non-derivatized and derivatized-DES/IDS were employed in order to obtain similar intensity of the precursor ions. MS/MS experiments were carried out in a Bruker HCT ultra ETD II ion trap (BrukerDaltonics, Bremen, Germany). Samples were infused at $1 \mu \mathrm{L} / \mathrm{min}$ using a $0.5 \mathrm{~mL}$ Hamilton syringe and a KdScientific syringe pump. The parameter settings for positive-ion ESI-MS were as follows: capillary voltage $-4000 \mathrm{~V}$; end plate offset $-500 \mathrm{~V}$; nebulizer $12 \mathrm{psi}$; drying gas $\left(\mathrm{N}_{2}\right) 5 \mathrm{~L} \times \mathrm{min}^{-1}$; drying gas temperature $300{ }^{\circ} \mathrm{C}$. The amplitude for CID MS/MS was 0.6. For ETD MS/MS, the maximum accumulation time of fluoranthene anions was $20 \mathrm{~ms}$. The "Remove" function was activated at $\mathrm{m} / \mathrm{z} 210$ to remove excessive fluoranthene anions. The reaction time was set to $100 \mathrm{~ms}$ and the cutoff was 160 . The ion charge control (ICC) ETD target was 500,000. "Smart Decomposition" was set at $\mathrm{z}=2$ or off. "Smart Decomposition" uses resonant excitation to dissociate fragment clusters that are held together by hydrogen bonds or other non-covalent forces after ETD without inducing further fragmentation. This function extends the reaction time automatically based on the $\mathrm{m} / \mathrm{z}$ ratio and charge state of the precursor ions. The software used for data processing and interpretation of the spectra was Compass 1.3 for Esquire HCT DataAnalysis (BrukerDaltonics).

\section{Results and Discussion}

\section{ETD Versus CID of Non-Derivatized DES and IDS}

DES and IDS are isomers consisting of a pyridinium ring where positions 3,4 , and 5 of the aromatic ring as well as the pyridinium nitrogen atom are substituted with amino acid chains of different length. The substituents at positions 3 and 5 are identical and contain two $\mathrm{CH}_{2}$ groups, while the substituents at position 4 (DES) or 2 (IDS) contain three $\mathrm{CH}_{2}$ groups, and the substituents at the pyridinium nitrogen atom contain four $\mathrm{CH}_{2}$ groups (Scheme 1a). Since DES and IDS show the same type of fragmentation upon ETD or CID, the patterns will be discussed using the results of DES (Figure 1). ETD of the doubly-charged precursor ion of nonderivatized DES $\left([\mathrm{DES}+\mathrm{H}]^{2+}, m / z\right.$ 263.6, Figure 1a) leads to the generation of two fragment ions derived from the loss of a neutral radical ([DES $\left.\left.-\mathrm{R}^{\circ}\right]^{+}, m / z 411.2\right)$ and a hydrogen atom ([DES] $]^{+}, m / z 526.3$ ) (the peak observed at $m / z 217$ is a background signal occurring in all spectra). The ETDderived, singly-charged fragment ion at $\mathrm{m} / \mathrm{z} 411.2$ ([DES $\left.\mathrm{R}^{*}\right]^{+}$) cannot be assigned unambiguously, since two pathways (I and II) may result in the loss of the same radical ( $\mathrm{R}^{\circ}$, Scheme 2). In order to elucidate which pathway is followed, the fragment ion at $m / z 411.2\left(\left[\mathrm{DES}-\mathrm{R}^{\circ}\right]^{+}\right)$was subjected to CID (Figure 1b), which led to the heterolytic N-C bond cleavage shown in Scheme 3. This cleavage derives in two fragment ions: one at $m / z 282.2\left(\left[\left[D E S-\mathrm{R}^{\circ}\right]-\mathrm{C}_{6} \mathrm{H}_{11} \mathrm{NO}_{2}\right]^{+}\right)$ and one at $m / z 130.1\left(\mathrm{C}_{6} \mathrm{H}_{12} \mathrm{NO}_{2}{ }^{+}\right)$corresponding to heterolytic $\mathrm{N}-\mathrm{C}$ bond cleavage with or without proton transfer, supported by intramolecular nucleophilic reaction with the amino group. This shows that pathway I in Scheme 2 is followed upon ETD to generate the $m / z 411.2$ fragment ion. We propose that ETD proceeds as follows: the doublycharged precursor ion ([DES $+\mathrm{H}]^{2+}, m / z$ 263.6, Scheme 2) captures an electron transferred from fluoranthene to one of the protonated primary amino groups, analogous to what has been described for peptides $[9,17,18]$, where it induces the transfer of a hydrogen atom to the electron-deficient pyridinium ring inducing the loss of a neutral radical $\left(\mathrm{R}^{\circ}, 116.0 \mathrm{Da}\right)$ triggered by the restoration of aromaticity resulting in $\left[\mathrm{DES}-\mathrm{R}^{*}\right]^{+}(\mathrm{m} / \mathrm{z}$ 411.2). A similar behavior has been observed in cyclohexadienones where single electron transfer was employed as a method of generating functionalized radicals that can subsequently cyclize or take part in cascade reaction processes [26]. The formation of [DES] ${ }^{+}(\mathrm{m} / \mathrm{z}$ 526.3 , Figure 1a) is explained by the loss of a hydrogen atom after electron transfer (Scheme 2). Proton donation from doubly charged DES to the fluoranthene radical anion may also be considered, but it is uncertain if the proton affinity of the fluoranthene radical anion $(\mathrm{m} / \mathrm{z} 202)$ is sufficiently high. The Bruker ion trap provides specific settings (not used by us) for proton transfer, where the ring-opened, monohydroanion derived from fluoranthene $(\mathrm{m} / \mathrm{z} 203)$ is used as an effective proton acceptor [31]. ETD was performed with and without smart decomposition giving identical results, which indicates that no $\mathrm{CID}$ is induced by the use of smart fragmentation.

CID of the doubly-charged precursor ion of DES ([DES + $\mathrm{H}^{2+}, m / z$ 263.7, Figure $\mathrm{S} 1$ in Supporting Information) leads to dissociation into a doubly charged product ion at $\mathrm{m} / \mathrm{z} 232.1$ $\left(\left[\mathrm{DES}-\mathrm{NH}_{3}-\mathrm{H}_{2} \mathrm{O}-\mathrm{CO}+\mathrm{H}\right]^{2+}\right)$ and two singly charged ions at $m / z 397.2\left(\left[\mathrm{DES}-\mathrm{C}_{6} \mathrm{H}_{11} \mathrm{NO}_{2}\right]^{+}\right)$, and at $\mathrm{m} / z 130.1$ $\left(\mathrm{C}_{6} \mathrm{H}_{12} \mathrm{NO}_{2}^{+}\right)$. The most comprehensive model currently available to describe how protonated molecules dissociate under CID conditions is based on the "mobile proton" hypothesis [32]. In this model, protonated molecules activated under lowenergy CID conditions fragment mainly by chargedirected reactions. Taking the DES structure into consideration, protonation likely takes place at one of the terminal primary amino groups where the proton can be stabilized by hydrogen bond formation with the adjacent carbonyl oxygen [33] (Scheme S1 in Supporting Information). Charge-directed reactions lead to the combined loss of 

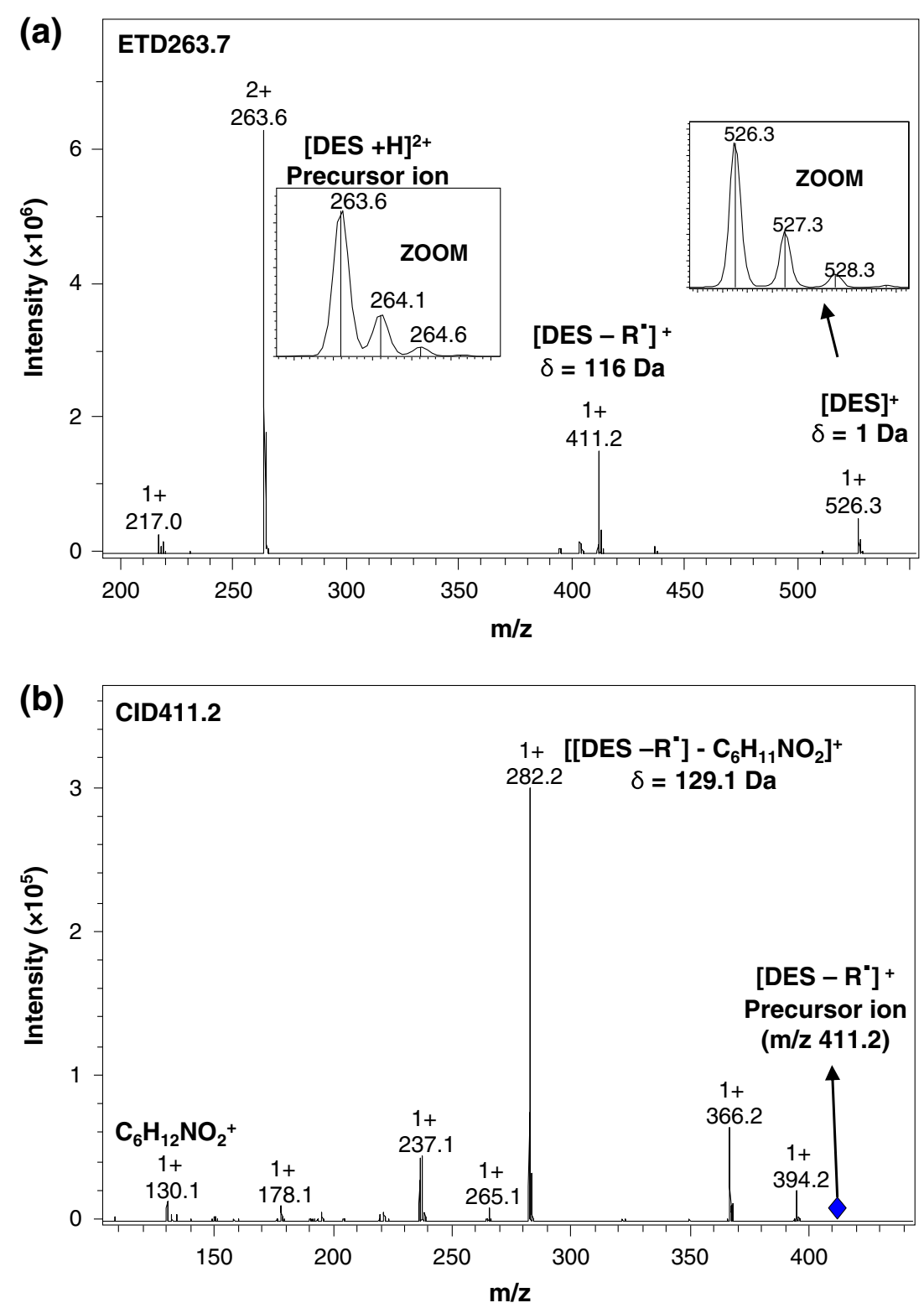

Figure 1. (a) Positive ESI-MS/MS in the ETD mode of doubly-charged DES $\left(\mathrm{m} / z\right.$ 263.6, $\left.[\mathrm{DES}+\mathrm{H}]^{2+}\right)$ upon infusion of a $0.05 \mathrm{mM}$ solution in $20 \%$ acetonitrile, $0.1 \%$ formic acid at $1 \mu \mathrm{L} / \mathrm{min}$. (b) Positive ESI-MS/MS in the CID mode of the singly-charged ETD fragment ion at $\mathrm{m} / z 411.2$ ([DES -R'] $]^{+}$, see (a)) upon infusion of $0.05 \mathrm{mM}$ DES in $20 \%$ acetonitrile, $0.1 \%$ formic acid at $1 \mu \mathrm{L} / \mathrm{min}$ (for ETD and CID conditions see experimental section)

small molecules $\left(\mathrm{NH}_{3}, \mathrm{H}_{2} \mathrm{O}\right.$, and $\mathrm{CO}$, doubly-charged fragment ion at $m / z 232.1$ ). In addition, the $\mathrm{N}-\mathrm{C}$ bond next to the nitrogen in the pyridinium ring undergoes heterolytic cleavage favored by the intramolecular nucleophilic reaction with the amino group (as already described in Scheme 3), yielding two singly charged product ions at $\mathrm{m} / \mathrm{z} 397.2\left(\left[\mathrm{DES}-\mathrm{C}_{6} \mathrm{H}_{11} \mathrm{NO}_{2}\right]^{+}\right)$, and at $\mathrm{m} / \mathrm{z}$ $130.1\left(\mathrm{C}_{6} \mathrm{H}_{12} \mathrm{NO}_{2}{ }^{+}\right)$.

\section{ETD Versus CID of DES Derivatized with Propionic Anhydride (PR-DES)}

Derivatization with propionic anhydride has been employed for the analysis of DES/IDS in urine. Derivatization facilitates the analysis by reversed-phase chromatography since it converts a highly hydrophilic analyte into a more hydrophobic compound that is retained on standard reversed phase LC columns [30]. Derivatization results in the conversion of primary amines into amide groups thus modifying the protonation site of the molecule, which may affect the ETD and/or CID patterns.

ETD resulted in a similar pattern as for non-derivatized DES (Figure S2 in Supporting Information) with respect to the generation of a fragment ion derived from the loss of a neutral radical (see $m / z 411.2$ ([DES $\left.-\mathrm{R}^{*}\right]^{+}$in Figure 1a for analogy). However, in this case, the fragment ion derived from the loss of a hydrogen atom after electron transfer was 


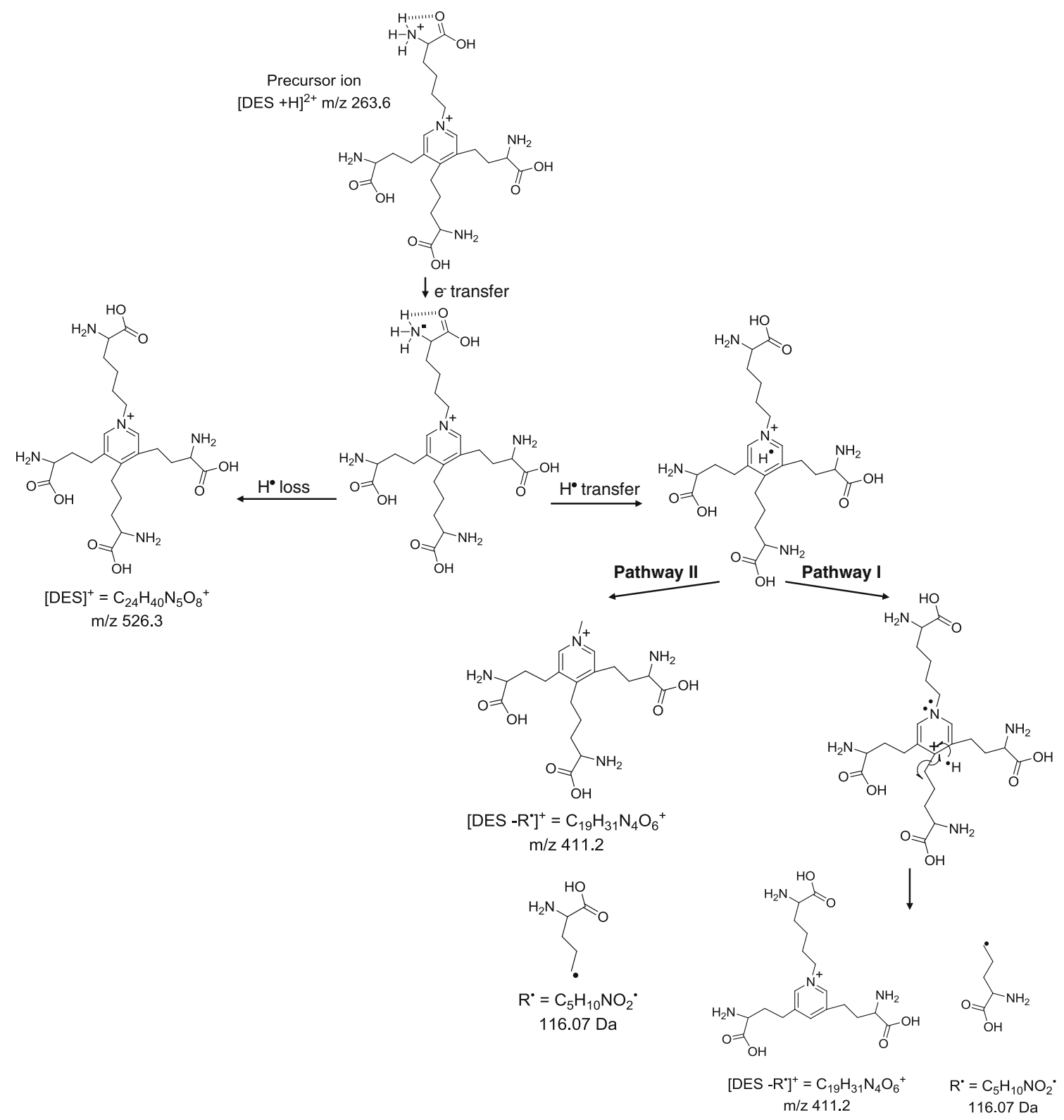

Scheme 2. ETD fragmentation pattern elucidation of doubly-charged DES $\left(m / z 263.6,[D E S+H]^{2+}\right)$

not detected. When ETD is performed on the doubly charged ion ([PR-DES $\left.+\mathrm{H}]^{2+}, m / z 375.7\right)$ we propose that an electron is transferred to the protonated carbonyl oxygen of the amide group (Scheme S2 in Supporting Information) in agreement with earlier reports on the proton affinities of methyl esters of $\mathrm{N}$-acetylated amino acids [33]. This intermediate is

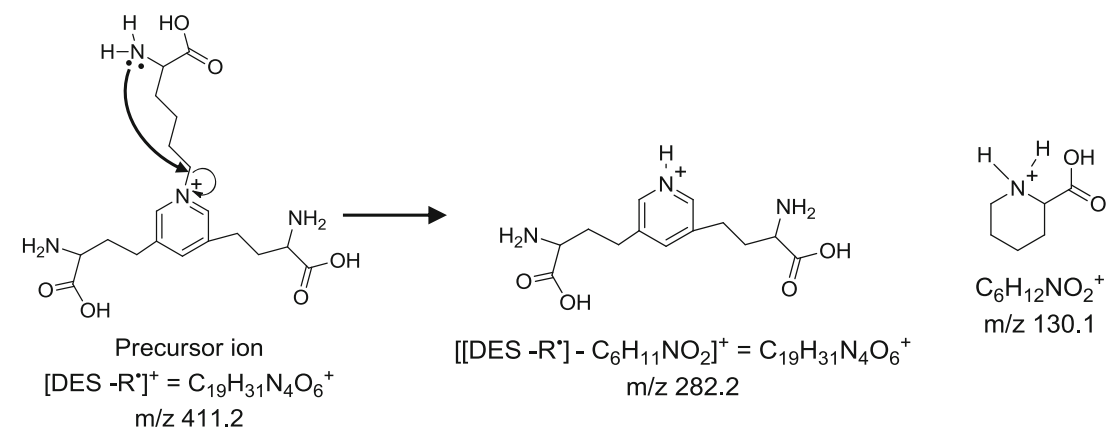

Scheme 3. CID fragmentation pattern elucidation of the singly-charged ETD fragment ion at $m / z 411.2\left(\left[D E S-R^{*}\right]^{+}\right.$, Figure 1a) 


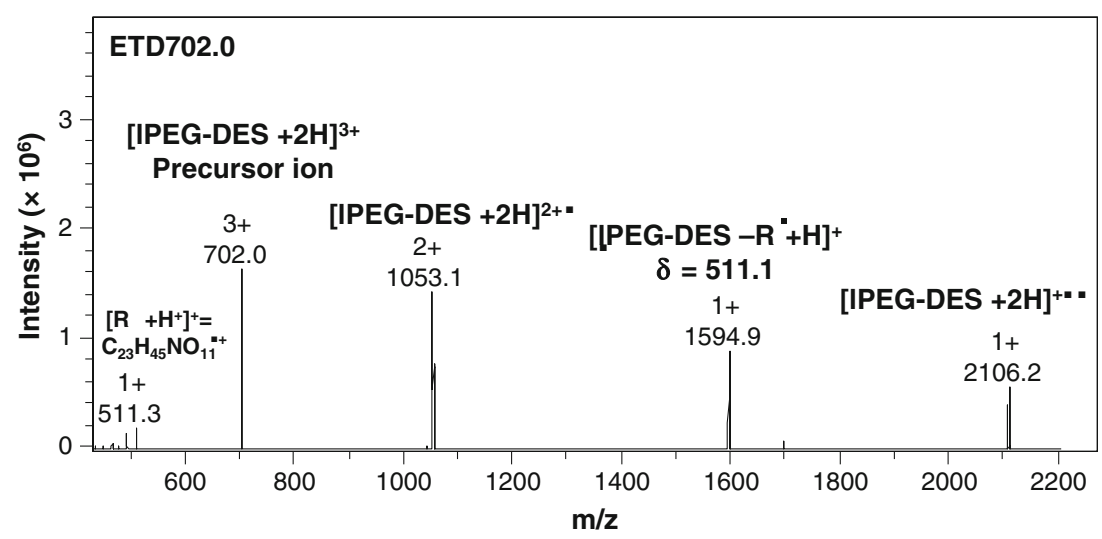

Figure 2. Positive ESI-MS/MS in the ETD mode of triply-charged longPEG-derivatized DES $\left(\mathrm{m} / \mathrm{z} 702.0\right.$, $\left.[\mathrm{IPEG}-\mathrm{DES}+2 \mathrm{H}]^{3+}\right)$ upon infusion of a $0.005 \mathrm{mM}$ solution in $20 \%$ acetonitrile, $0.1 \%$ formic acid at $1 \mu \mathrm{L} / \mathrm{min}$ (for ETD conditions see experimental section)

stabilized through the transfer of a hydrogen atom to the pyridinium ring leading to the loss of a $\mathrm{C}_{8} \mathrm{H}_{14} \mathrm{NO}_{3}$ radical and formation of a product ion at $\mathrm{m} / \mathrm{z} 579.3$ in analogy to non-derivatized DES (pathway I, Scheme 2) and as described for peptides [9, 17, 18].

Contrary to ETD, the CID pattern of PR-DES differs from that of the non-derivatized molecule. CID on PR-DES leads to the loss of $\mathrm{H}_{2} \mathrm{O}, \mathrm{CO}$, and $\mathrm{C}_{3} \mathrm{H}_{4} \mathrm{O}$ (56 Da) (Figure $\mathrm{S} 3$ and Scheme $\mathrm{S} 3$ in Supporting Information). Since derivatization with propionic anhydride converts the primary amine into an amide, protonation will not take place at the nitrogen atom but rather at the carbonyl oxygen, as outlined above [33]. Collision induced dissociation will therefore result in the loss of $\mathrm{H}_{2} \mathrm{O}$, $\mathrm{CO}$ and $\mathrm{C}_{3} \mathrm{H}_{4} \mathrm{O}\left(\left[\mathrm{PR}-\mathrm{DES}-\mathrm{C}_{3} \mathrm{H}_{4} \mathrm{O}\right]^{2+}, 56 \mathrm{Da}, \mathrm{m} / z\right.$ 347.7) through charge directed fragmentation in an analogous manner as the observed loss of ketene from acetylated amino acids and peptides. The loss of a side chain in analogy to the mechanism given in Scheme 3 does not take place.

\section{ETD Versus CID of DES Derivatized with PEG Molecules of Different Length}

PEG is widely used in the derivatization of proteins and peptides notably to extend their in vivo half-lives [34]. We recently showed that $\mathrm{PEG}$ derivatization may also be useful in enhancing the ionization efficiency of small molecules such as amino acids, as well as to increase their hydrophobicity allowing analysis by reversed-phase LC-MS [29]. In addition, PEG ether functionalities may be protonated which could affect the ETD and CID pattern of derivatized DES $[19,35,36]$. To investigate the behavior upon ETD and CID, DES was derivatized with PEG molecules consisting of 1 (shortPEG), 4 (mediumPEG), or 7 (longPEG) ethylene glycol units (Scheme 1b). In addition, these molecules differ slightly in their structure. While short and longPEG have two $\mathrm{CH}_{2}$ groups between the carbonyl group and the first ether oxygen atom of the PEG chain, mediumPEG has only one $\mathrm{CH}_{2}$ group. After derivatization, shortPEG-DES and mediumPEG-DES were detected mainly as doubly charged ions while longPEG-DES was primarily detected as triply charged ion.

ETD of doubly charged shortPEG-DES and triplycharged longPEG-DES resulted in similar patterns as for non-derivatized-DES and PR-DES with the loss a radical and a proton through pathway I (see Figure 2 and Scheme 4 for longPEG-DES as an example). Protonation in pegylatedDES most probably takes places at the carbonyl group of the PEG molecule and in the case of longPEG-DES an extra proton may be located in the PEG-chain itself (Scheme 4) due to stabilization by hydrogen bond formation with the

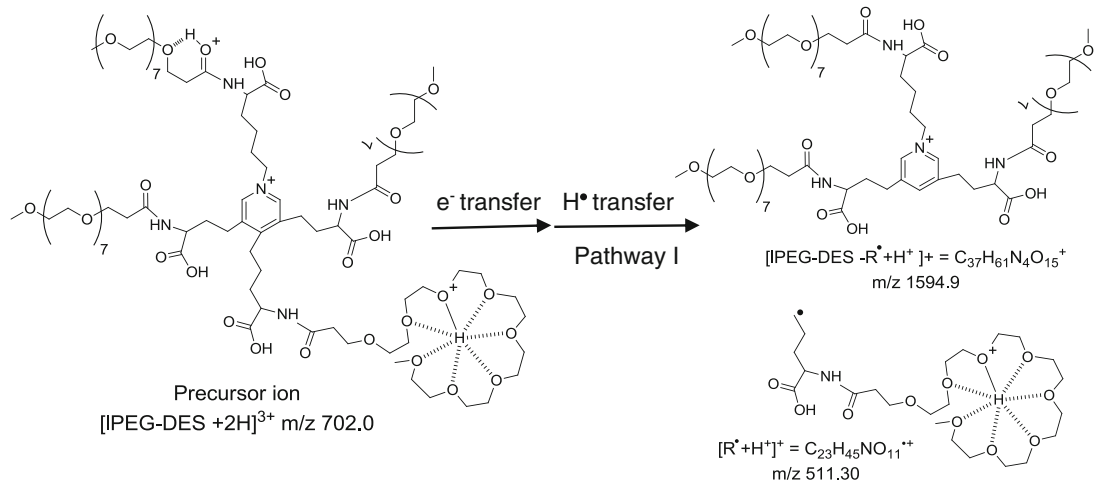

Scheme 4. ETD fragmentation pattern elucidation of triply-charged longPEG-derivatized DES $\left(\mathrm{m} / z\right.$ 702.0, $\left.[\mathrm{IPEG}-\mathrm{DES}+2 \mathrm{H}]^{3+}\right)$ 

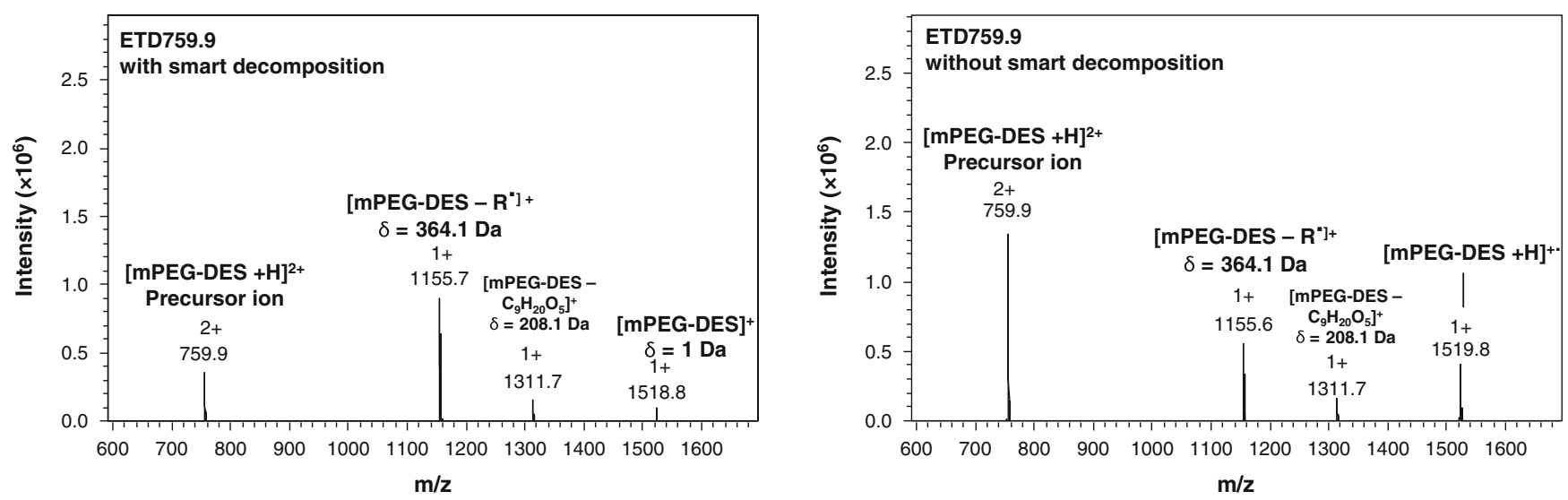

Figure 3. Positive ESI-MS/MS in the ETD mode (left: with smart decomposition; right: without smart decomposition) of doubly-charged mediumPEG-derivatized DES $\left.(\mathrm{m} / \mathrm{z} \text { 759.9, [mPEG-DES }+\mathrm{H}]^{2+}\right)$ upon infusion of a $0.005 \mathrm{mM}$ solution in $20 \%$ acetonitrile, $0.1 \%$ formic acid at $1 \mu \mathrm{L} / \mathrm{min}$ (for ETD conditions see experimental section)

PEG chain ether oxygen atoms [37]. The assumption that an extra proton is located within the side chain that is lost from the longPEG-DES is corroborated by detection of the protonated radical at $\mathrm{m} / \mathrm{z} 511.3$ that is generated through pathway I $\left(\mathrm{C}_{23} \mathrm{H}_{45} \mathrm{NO}_{11}{ }^{+}\right.$, Figure 2).

ETD of mediumPEG-DES resulted in the generation of an additional fragment ion ([mPEG-DES $\left.-\mathrm{C}_{9} \mathrm{H}_{20} \mathrm{O}_{5}\right]^{+}, \mathrm{m} / \mathrm{z}$ 1311.7, Figure 3) apart from the already described loss of a radical through pathway I ([mPEG-DES $\left.-\mathrm{R}^{\circ}\right]^{+}, 364.1 \mathrm{Da}$, Figure 3). Generation of [mPEG-DES $\left.-\mathrm{C}_{9} \mathrm{H}_{20} \mathrm{O}_{5}\right]^{+}$may be explained by the fact that an electron is transferred from fluoranthene to the protonated carbonyl group from the PEG molecule where it is stabilized along the five-membered ring that is formed by hydrogen bonding within the PEG molecule inducing its fragmentation (Scheme 5). This fragmentation only takes place in mediumPEG-DES and not in short or longPEGDES most likely due to the difference in structures (number of $\mathrm{CH}_{2}$ units between the carbonyl and the first ether group within the PEG chain, Scheme 1b). Stabilization of the electron along the five-membered ring in the PEG molecule is supported by the observation that the singly charged mediumPEG-DES radical ion $\left([\mathrm{mPEG}-\mathrm{DES}+\mathrm{H}]^{+*}, m / z 1519.8\right.$, Figure 3 right spectrum) is detected when no smart decomposition is employed. The use of resonant excitation during smart decomposition favors the loss ([mPEG-DES]+, $m / z$ 1518.8, Figure 3 left spectrum) and transfer of a hydrogen atom to the pyridinium ring to induce the loss of a radical following pathway I ([mPEG-DES $\left.-\mathrm{R}^{\circ}\right]+$, $\mathrm{m} / \mathrm{z} 1155.7$, Scheme 5) as previously described for the non-, and propionic anhydride-derivatized DES.

To confirm the structure of $\mathrm{C}_{9} \mathrm{H}_{20} \mathrm{O}_{5}$, ETD was performed on doubly charged mediumPEG-DES that was derivatized with mediumPEG containing 0 or $5{ }^{13} \mathrm{C}$ atoms (Scheme 5 for the location of the ${ }^{13} \mathrm{C}$ atoms within the PEG molecule). When DES is derivatized with unlabeledmediumPEG a loss of 208.1 Da is observed (loss of $\mathrm{C}_{9} \mathrm{H}_{20} \mathrm{O}_{5}, m / z$ 1311.7, Figure 3) while a loss of 212.1 Da is observed when DES is derivatized with ${ }^{13} \mathrm{C}_{5}$-mediumPEG. The $4 \mathrm{Da}$ mass difference is in agreement with the proposed fragmentation pattern (Scheme 5).

CID of DES derivatized with the different PEG molecules shows that it follows a charge-directed mechanism (see Figure 4 for an example of the mediumPEG-DES CID spectrum). Figure 4 shows that fragmentation takes place by the loss of $\mathrm{H}_{2} \mathrm{O}, \mathrm{CO}$, and $\mathrm{C}_{7} \mathrm{H}_{16} \mathrm{O}_{4}\left(\left[\text { PEG-DES }-\mathrm{C}_{7} \mathrm{H}_{16} \mathrm{O}_{4}+\mathrm{H}\right]^{2+}, 164.1 \mathrm{Da}, \mathrm{m} / \mathrm{z}\right.$ 677.8) which was confirmed by $\mathrm{CID}$ of DES that was derivatized with unlabeled-mediumPEG and ${ }^{13} \mathrm{C}_{5}$-mediumPEG-OPFP. The observed fragments can be explained by the fact that protonation takes places at the PEG carbonyl oxygen atom where the proton is stabilized by hydrogen bond formation between the carbonyl group and one of the oxygen-ether atoms of the PEG molecule

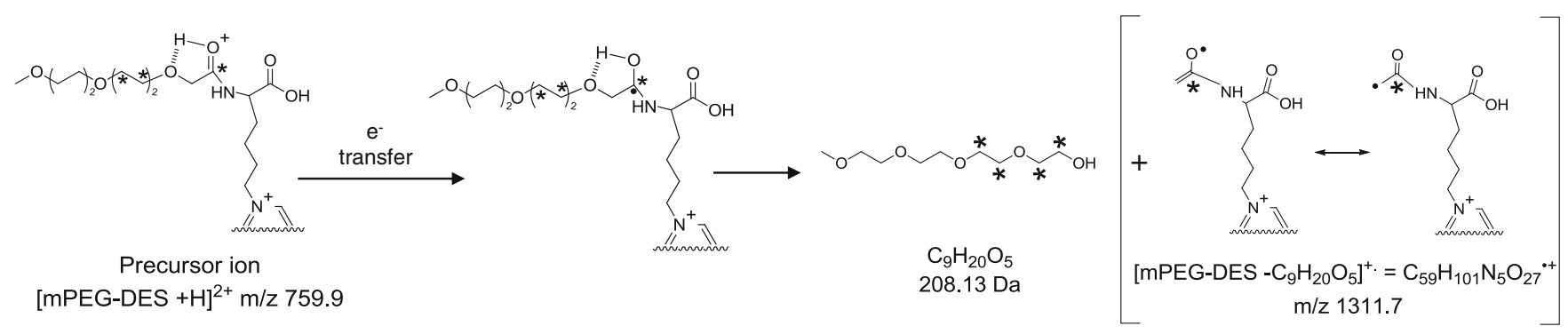

Scheme 5. ETD Fragmentation pattern elucidation of doubly-charged mediumPEG-derivatized DES $(\mathrm{m} / \mathrm{z}$ 759.9, [mPEG-DES $+\mathrm{H}]^{2+}$ ). Location of the ${ }^{13} \mathrm{C}$ atoms within the PEG molecule is marked with * 


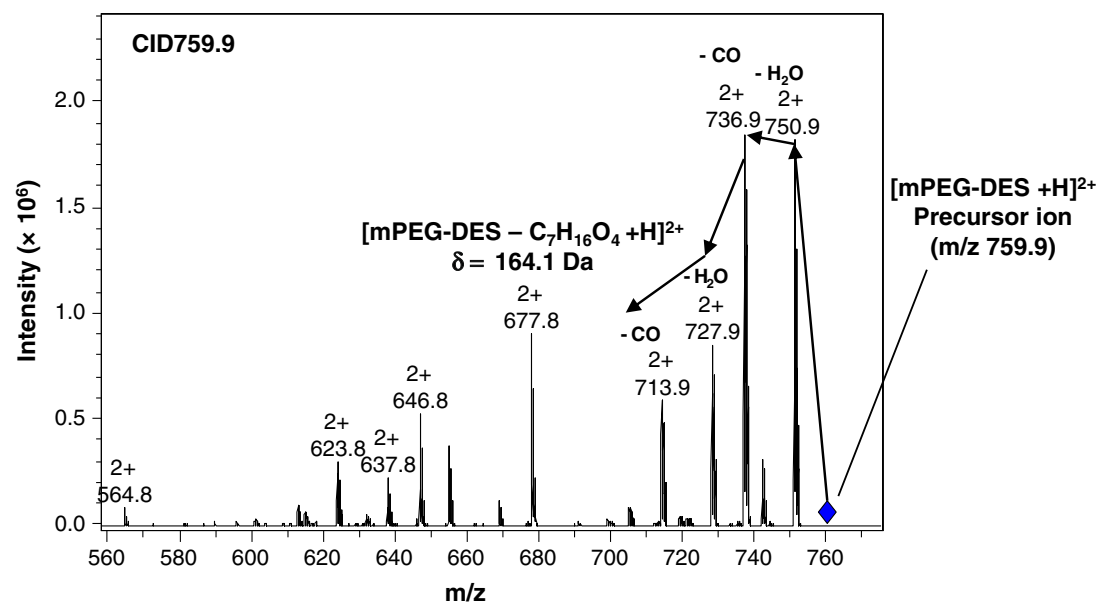

Figure 4. Positive ESI-MS/MS in the CID mode of doubly-charged mediumPEG-derivatized DES $\left(\mathrm{m} / \mathrm{z}\right.$ 759.9, $\left.[\mathrm{mPEG}-\mathrm{DES}+\mathrm{H}]^{2+}\right)$ upon infusion of a $0.005 \mathrm{mM}$ solution in $20 \%$ acetonitrile, $0.1 \%$ formic acid at $1 \mu \mathrm{L} / \mathrm{min}$ (for CID conditions see experimental section)

(Scheme 6a). Due to the difference in structure, hydrogen bond formation within shortPEG-DES after protonation resulted in the formation of fragment ions through loss of $\mathrm{H}_{2} \mathrm{O}, \mathrm{CO}$, and $\mathrm{C}_{3} \mathrm{H}_{8} \mathrm{O}_{2}$ (76 Da) (Scheme 6b). The CID spectra of longPEGDES resulted in a very complex spectrum (results not shown) where characteristic losses of ethylene oxide units, as commonly observed in the mass spectra of PEG-containing surfactants and free PEG molecules, dominated the CID pattern [36]. This is because longPEG-DES contrary to shortPEG-DES and mediumPEG-DES can accommodate an extra proton within the PEG chain triggering the typical CID fragmentation of PEG molecules $[19,36]$.

Previous ECD studies on free PEG molecules reported a similar fragmentation pattern as in CID (loss of ethylene oxide units, $44 \mathrm{Da}$ ) [14, 30]. This type of fragmentation was, however, not observed for any of the studied DES-PEG derivatives upon ETD. The absence of this type of fragmentation indicates that the hydrogen atom that is generated after electron transfer is not transferred to the ether functionalities of the PEG molecule [14, 30], but to the electron deficient pyridinium ring, which acts as a "hydrogen atom trap."

\section{Conclusions}

DES and IDS are molecules of interest in the study of diseases that progress through elastin breakdown. Due to their molecular structure they are detectable as doubly charged ions in ESI-MS. This allows the comparison of ETD and CID mechanisms in other molecules than peptides, providing a more detailed understanding of ETD pathways. DES and IDS were shown to fragment similarly in both fragmentation modes, since the amino acid side chains (chain in position 4 in DES and 2 in IDS, Scheme 1a) occupy equivalent positions in the pyridinium ring (position 4 and 2 are both low electron density positions) and do not affect the initial protonation site.

Clear differences in the CID and ETD patterns/mechanisms were observed. Since CID is driven by chargedirected reactions, fragmentation is mainly determined by the initial protonation site(s) and by the hydrogen bond interactions of the proton within the molecule. CID patterns are, therefore, affected by derivatizations that modify the protonation site(s) or hydrogen bonding of the proton within (a)

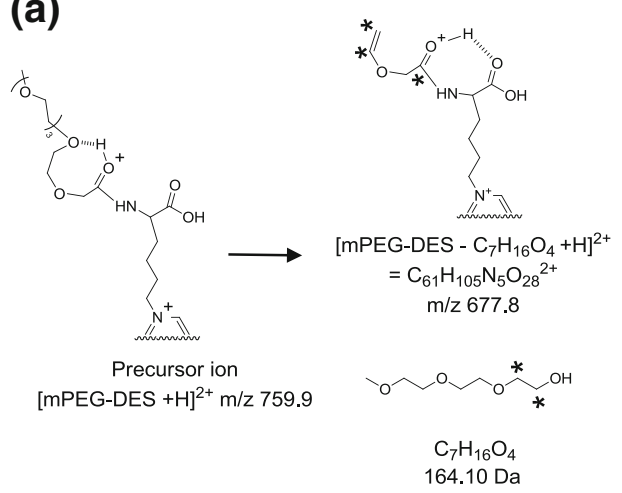

(b)

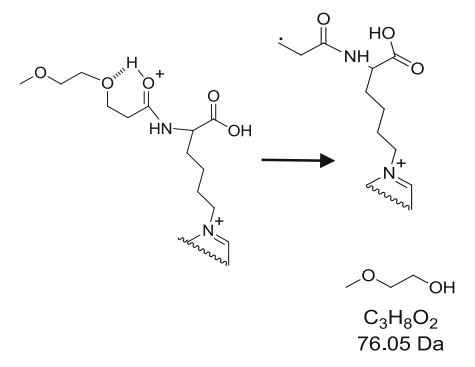

Scheme 6. CID fragmentation pattern elucidation of doubly-charged (a) mediumPEG-DES, (b) shortPEG-DES 
the molecule. On the other hand, ETD is induced and determined by the generation of a hydrogen atom and its transfer to electron deficient groups within the molecule. ETD of derivatized DES/IDS is dominated by the effect of electron deficient groups in the molecule (such as the pyridinium ring in DES/IDS) and is thus not affected by derivatization.

A practical advantage of ETD is its simpler fragmentation when compared to CID in the case of DES/IDS. While CID spectra consist of many non-informative fragment ions due to the loss of $\mathrm{H}_{2} \mathrm{O}, \mathrm{NH}_{3}$, or CO, ETD spectra consist of 1-2 major fragment ions that are derived from the loss of characteristic side-chain radicals. We are currently investigating whether ETD can be used for the sensitive, targeted analysis of DES/ IDS in biological samples from COPD patients.

\section{Acknowledgments}

The authors acknowledge support for this project by grants from the University of Groningen and The Netherlands Proteomics Center.

\section{References}

1. Sleno, L., Volmer, D.A.: Ion activation methods for tandem mass spectrometry. J. Mass Spectrom. 39, 1091-1112 (2004)

2. Wysocki, V.H., Jones, C.M., Galhena, A.S., Blackwell, A.E.: Surfaceinduced dissociation shows potential to be more informative than collision-induced dissociation for structural studies of large systems. $J$. Am. Soc. Mass Spectrom. 19, 903-913 (2008)

3. Viet Hung, N., Afonso, C., Tabet, J.-C.: Comparison of collisioninduced dissociation and electron-induced dissociation of singly charged mononucleotides. Int. J. Mass Spectrom. 316, 140-146 (2012)

4. Feketeova, L., Khairallah, G.N., Brunet, C., Lemoine, J., Antoine, R., Dugourd, P., O'Hair, R.A.J.: Fragmentation of the tryptophan cluster $[\operatorname{Trp}(9)-2 \mathrm{H}](2-)$ induced by different activation methods. Rapid Commun. Mass Spectrom. 24, 3255-3260 (2010)

5. Madsen, J.A., Brodbelt, J.S.: Comparison of infrared multiphoton dissociation and collision-induced dissociation of supercharged peptides in ion traps. J. Am. Soc. Mass Spectrom. 20, 349-358 (2009)

6. Zubarev, R.A., Kelleher, N.L., McLafferty, F.W.: Electron capture dissociation of multiply charged protein cations. A nonergodic process. J. Am. Chem. Soc. 120, 3265-3266 (1998)

7. Syka, J.E.P., Coon, J.J., Schroeder, M.J., Shabanowitz, J., Hunt, D.F.: Peptide and protein sequence analysis by electron transfer dissociation mass spectrometry. Proc. Natl. Acad. Sci. U.S.A. 101, 9528-9533 (2004)

8. McLafferty, F.W., Bryce, T.A.: Metastable-ion characteristics - characterization of isomeric molecules. Chem. Comm. 1215-1217 (1967)

9. Simons, J.: Analytical model for rates of electron attachment and intramolecular electron transfer in electron transfer dissociation mass spectrometry. J. Am. Chem. Soc. 132, 7074-7085 (2010)

10. Zubarev, R.A.: Electron-capture dissociation tandem mass spectrometry. Curr. Opin. Biotechnol. 15, 12-16 (2004)

11. Jones, A.W., Cooper, H.J.: Dissociation techniques in mass spectrometrybased proteomics. Analyst 136, 3419-3429 (2011)

12. Wiesner, J., Premsler, T., Sickmann, A.: Application of electron transfer dissociation (ETD) for the analysis of posttranslational modifications. Proteomics 8, 4466-4483 (2008)

13. Mikesh, L.M., Ueberheide, B., Chi, A., Coon, J.J., Syka, J.E.P., Shabanowitz, J., Hunt, D.F.: The utility of ETD mass spectrometry in proteomic analysis. Biochim. Biophys. Acta 1764, 1811-1822 (2006)

14. Sohn, C.H., Chung, C.K., Yin, S., Ramachandran, P., Loo, J.A., Beauchamp, J.L.: Probing the mechanism of electron capture and electron transfer dissociation using tags with variable electron affinity. J. Am. Chem. Soc. 131, 5444-5459 (2009)
15. Zubarev, R.A., Haselmann, K.F., Budnik, B., Kjeldsen, F., Jensen, F.: Towards an understanding of the mechanism of electron-capture dissociation: a historical perspective and modern ideas. Eur. J. Mass Spectrom. 8, 337-349 (2002)

16. Zubarev, R.A., Zubarev, A.R., Savitski, M.M.: Electron capture/transfer versus collisionally activated/induced dissociations: Solo or duet? $J$. Am. Soc. Mass Spectrom. 19, 753-761 (2008)

17. Simons, J.: Mechanisms for S-S and $\mathrm{N}-\mathrm{C}(\alpha)$ bond cleavage in peptide ECD and ETD mass spectrometry. Chem. Phys. Lett. 484, 81-95 (2010)

18. Neff, D., Smuczynska, S., Simons, J.: Electron shuttling in electron transfer dissociation. Int. J. Mass Spectrom. 283, 122-134 (2009)

19. Cerda, B.A., Breuker, K., Horn, D.M., McLafferty, F.W.: Charge/ radical site initiation versus coulombic repulsion for cleavage of multiply charged ions. Charge solvation in poly(alkene glycol) ions. $J$. Am. Soc. Mass Spectrom. 12, 565-570 (2001)

20. Hakansson, K., Hudgins, R.R., Marshall, A.G., O'Hair, R.A.J.: Electron capture dissociation and infrared multiphoton dissociation of oligodeoxynucleotide dications. J. Am. Soc. Mass Spectrom. 14, 23-41 (2003)

21. Liu, H., Yoo, H.J., Hakansson, K.: Characterization of phosphatecontaining metabolites by calcium adduction and electron capture dissociation. J. Am. Soc. Mass Spectrom. 19, 799-808 (2008)

22. Han, L., Costello, C.E.: Electron transfer dissociation of milk oligosaccharides. J. Am. Soc. Mass Spectrom. 22, 997-1013 (2011)

23. Liang, X., Liu, J., Leblanc, Y., Covey, T., Ptak, A.C., Brenna, J.T., McLuckey, S.A.: Electron transfer dissociation of doubly sodiated glycerophosphocholine lipids. J. Am. Soc. Mass Spectrom. 18, 17831788 (2007)

24. Partridge, S.M., Thomas, J., Elsden, D.F.: Constitution of CrossLinkages in Elastin. Nature 197, 1297-1298 (1963)

25. Viglio, S., Annovazzi, L., Luisetti, M., Stolk, J., Casado, B., Iadarola, P.: Progress in the methodological strategies for the detection in real samples of desmosine and isodesmosine, two biological markers of elastin degradation. J. Sep. Sci. 30, 202-213 (2007)

26. Ma, S., Turino, G.M., Lin, Y.Y.: Quantitation of desmosine and isodesmosine in urine, plasma, and sputum by LC-MS/MS as biomarkers for elastin degradation. J. Chromatogr. B 879, 1893-1898 (2011)

27. Ma, S.R., Lin, Y.Y., Tartell, L., Turino, G.M.: The effect of tiotropium therapy on markers of elastin degradation in COPD. Resp. Res. 10:12 (2009)

28. Luisetti, M., Ma, S., Iadarola, P., Stone, P.J., Viglio, S., Casado, B., Lin, Y.Y., Snider, G.L., Turino, G.M.: Desmosine as a biomarker of elastin degradation in COPD: current status and future directions. Eur. Respir. J. 32, 1146-1157 (2008)

29. Abello, N., Geurink, P.P., van der Toorn, M., van Oosterhout, A.J.M., Lugtenburg, J., van der Marel, G.A., Kerstjens, H.A.M., Postma, D.S., Overkleeft, H.S., Bischoff, R.: Poly(ethylene glycol)-based stable isotope labeling reagents for the quantitative analysis of low molecular weight metabolites by LC-MS. Anal. Chem. 80, 9171-9180 (2008)

30. Boutin, M., Berthelette, C., Gervais, F.G., Scholand, M.B., Hoidal, J., Leppert, M.F., Bateman, K.P., Thibault, P.: High-sensitivity nanoLCMS/MS analysis of urinary desmosine and isodesmosine. Anal. Chem. 81, 1881-1887 (2009)

31. Hartmer, R., Kaplan, D.A., Gebhardt, C.R., Ledertheil, T., Brekenfeld, A.: Multiple ion/ion reactions in the 3D ion trap: selective reagent anion production for ETD and PTR from a single compound. Int. J. Mass Spectrom. 276, 82-90 (2008)

32. Paizs, B., Suhai, S.: Fragmentation pathways of protonated peptides. Mass Spectrom. Rev. 24, 508-548 (2005)

33. Addario, V., Guo, Y.Z., Chu, I.K., Ling, Y., Ruggerio, G., Rodriquez, C.F., Hopkinson, A.C., Siu, K.W.M.: Proton affinities of methyl esters of Nacetylated amino acids. Int. J. Mass Spectrom. 219, 101-114 (2002)

34. Roberts, M.J., Bentley, M.D., Harris, J.M.: Chemistry for peptide and protein PEGylation. Adv. Drug Deliv. Rev. 54, 459-476 (2002)

35. Cerda, B.A., Horn, D.M., Breuker, K., Carpenter, B.K., McLafferty, F.W.: Electron capture dissociation of multiply-charged oxygenated cations. A nonergodic process. Eur. Mass Spectrom. 5, 335-338 (1999)

36. Lattimer, R.P.: Tandem mass-spectrometry of poly(ethylene glycol) proton-attachment and deuteron-attachment ions. Int. J. Mass Spectrom. Ion Process. 116, 23-36 (1992)

37. Gidden, J., Wyttenbach, T., Jackson, A.T., Scrivens, J.H., Bowers, M.T.: Gas-phase conformations of synthetic polymers: Poly(ethylene glycol), poly(propylene glycol), and poly(tetramethylene glycol). J. Am. Chem. Soc. 122, 4692-4699 (2000) 